\title{
Expression profiling and long lasting responses to chemotherapy in metastatic gastric cancer
}

\author{
CRISTIANA LO NIGRO ${ }^{1}$, MARTINO MONTEVERDE ${ }^{1}$, MICHELA RIBA $^{1}$, LAURA LATTANZIO ${ }^{1}$, \\ FEDERICA TONISSI $^{1}$, ORNELLA GARRONE ${ }^{1}$, ABDELHAMID HEOUAINE $^{1}$, FABIO GALLO $^{2}$, \\ MARCELLO CEPPI $^{2}$, FELICE BORGHI $^{3}$, ALBERTO COMINO $^{4}$ and MARCO MERLANO ${ }^{1}$
}

\author{
${ }^{1}$ Laboratorio di Oncologia Translazionale, Oncologia, S. Croce General Hospital, via Carle 25, I-12100 Cuneo; \\ ${ }^{2}$ Molecular Epidemiology, National Cancer Research Institute, L.go Benzi 10, I-16132 Genova; ${ }^{3}$ Chirurgia Generale, \\ S. Croce General Hospital; ${ }^{4}$ Anatomia Patologica, S. Croce General Hospital, via Coppino 26, I-12100 Cuneo, Italy
}

Received May 27, 2010; Accepted July 5, 2010

DOI: 10.3892/ijo_00000773

\begin{abstract}
Current palliative chemotherapy (CT) regimens achieve clinical benefits in less than $50 \%$ of patients treated for metastatic gastric cancers, and long-term survivals are anecdotical. Genetic polymorphisms and differences at the level of transcription in genes involved in biological processes of drug metabolism, DNA repair and drug resistance can explain the observed individual differences in response to drugs, in survival and in different susceptibility to the toxic effects of CT. The possibility to classify patients on the basis of genetic signatures could help in choosing the CT regimen. We present herein an analysis of genetic and expression profiling of three patients affected by metastatic gastric cancer, treated with CT and alive, disease-free, at 66-82 months. Four patients with typical clinical outcome represented the control group. Expression profiling from paraffin-embedded tumor tissues was performed on an ad hoc set of genes involved in drug metabolism and resistance, DNA repair, cell cycle regulation and growth factors signalling. Genetic polymorphism analysis on DNA extracted from peripheral blood was done by pyrosequencing of genetic markers predictive of drug response. Expression analysis in long-term survivors revealed a significant upregulation of PTEN, TP63, GADD45a and MAPK1 genes. We found also an upregulation of CYP1A1, CYP3A4 and ERBB4 genes. EGF was found to be down-regulated in long-term survivors. ERCC1 C8092A polymorphism seems to be associated with survival in our set of patients. The present study shed light on a set of genes, which could have a predictive role in survival of patients with metastatic gastric tumors.
\end{abstract}

Correspondence to: Dr Cristiana Lo Nigro, Laboratorio di Oncologia Translazionale, Oncologia, S. Croce General Hospital, Via Carle 25, I-12100 Cuneo, Italy

E-mail: lonigro.c@ospedale.cuneo.it

Key words: metastatic gastric cancer, chemotherapy, expression profiling, single nucleotide polymorphisms, survival

\section{Introduction}

Gastric cancer is among the most frequent malignancies, it accounts for roughly 800,000 new cases every year worldwide and represents the second cause of cancer death (1). It is a heterogeneous disease both histologically and genetically, and patient outcome is difficult to predict using classical histological and molecular classification.

In spite of the great therapeutic enhancements, many patients treated for gastric cancer do not reach recovery. Disease is often diagnosed in advanced stages and is therefore associated with poor prognosis. Current palliative chemotherapy (CT) regimens achieve clinical benefits in less than $50 \%$ of patients treated for metastatic gastric cancers, and long-term survivals are rare (2).

The significant survival benefit of 5-fluorouracil (5-FU)based CT for metastatic gastric cancer compared with best supportive care has been reported (3). In Western countries, 5-FU combined with cisplatin, plus/minus epirubicin, have been referred to as the standard chemotherapy for metastatic gastric cancer, with the median survival time ranging from 7.3 to 10.5 months (3). Combination CT of those drugs has increased response rates to over $50 \%$, but has not increased the median survival time to over 11 months, and the therapies are often related to a higher frequency of severe adverse events than older regimens (3). Thus, there is a need for diagnostic methods that allow the prediction of clinical outcome and enable the pre-therapeutic discrimination of treatment effect.

Currently, there are no efficient clinical, pathological or molecular markers to distinguish between responders and non-responders, and between long and normal survivors, in relation to chemotherapy regimens used in gastric cancer (4).

A growing body of evidence suggests that the intratumor protein expression, single nucleotide polymorphisms and gene expression of drug-metabolizing enzymes, DNA repair enzymes, or angiogenic enzymes may have important implications for anticancer drug efficacy. Few studies have investigated the predictive or prognostic value of these genetic markers in patients with advanced gastric cancer (4). 
Table I. Clinical characteristics and survival of the patients. ${ }^{a}$

\begin{tabular}{|c|c|c|c|c|c|c|c|c|c|c|c|}
\hline No. & $\begin{array}{c}\text { Date of } \\
\text { diagnosis }\end{array}$ & $\begin{array}{l}\text { Tumor } \\
\text { type }\end{array}$ & $\begin{array}{c}\text { Site of } \\
\text { metastasis }\end{array}$ & Treatment & $\begin{array}{c}\text { Start } \\
\text { therapy }\end{array}$ & $\begin{array}{c}\text { End } \\
\text { therapy }\end{array}$ & $\begin{array}{c}\text { Last } \\
\text { follow-up }\end{array}$ & Status & $\begin{array}{c}\text { PFS } \\
\text { (months) }\end{array}$ & $\begin{array}{c}\text { Long/ } \\
\text { normal } \\
\text { survivor }\end{array}$ & $\begin{array}{c}\text { Median } \\
\text { survival } \\
\text { (months) }\end{array}$ \\
\hline
\end{tabular}

\begin{tabular}{|c|c|c|c|c|c|c|c|c|c|c|}
\hline $02 / 2004$ & Gastric & Liver & $\mathrm{ECF} \times 8$ & $03 / 04$ & 08/2004 & $01 / 2010$ & NED & 66 & Long & \\
\hline $08 / 2004$ & Gastric & Liver & ECF $\times 6$ & $09 / 03$ & $12 / 2003$ & $11 / 2009$ & NED & 75 & Long & 75 \\
\hline $05 / 2003$ & Cardias & $\begin{array}{l}\text { Non-regional } \\
\text { nodes }\end{array}$ & $\begin{array}{l}\text { TCF x } 4 \text { followed } \\
\text { by surgery due to } \\
\text { the observed CR }\end{array}$ & $06 / 03$ & 09/2003 & 03/2010 & NED & 82 & Long & \\
\hline $10 / 2000$ & Gastric & Liver & ECF x 6 & $09 / 05$ & $01 / 2006$ & $11 / 2006$ & Exitus & 14 & Normal & \\
\hline $03 / 2001$ & Gastric & Liver & ECF $\times 6$ & 04/01 & $08 / 2001$ & $05 / 2004$ & Exitus & 33 & Normal & \\
\hline $08 / 2001$ & Gastric & $\begin{array}{c}\text { Liver and } \\
\text { lymph nodes }\end{array}$ & ECF $x 6$ & 08/01 & $01 / 2002$ & $05 / 2002$ & Exitus & 9 & Normal & 13 \\
\hline $02 / 2002$ & Gastric & Liver and cns & ECF x 6 & $02 / 02$ & $06 / 2002$ & $02 / 2003$ & Exitus & 12 & Normal & \\
\hline
\end{tabular}

${ }^{\mathrm{a} E C F}$, epirubicin, cisplatin and 5-FU; TCF, taxanes, cisplatin and 5-FU; cns, central nervous system; NED, no evidence of disease; PFS, progression-free survival; $\mathrm{CR}$, complete response.

Here, we report on a long lasting response to epirubicin/ cisplatin/5-FU combination CT in 3 metastatic gastric cancer patients. We further provide information on gene expression profiling and genetic analysis demonstrating its potential as a predictor for survival in metastatic gastric cancer.

\section{Patients and methods}

Patients. In the clinical practice of the Medical Oncology Department of S. Croce General Hospital, 3 long-term survival cases with metastatic gastric cancer after chemotherapy, who were treated with a standard ECF regimen (epirubicin, cisplatin, 5-FU) and have shown prolonged disease-free and overall survival, have been observed. The 3 patients had histologically confirmed metastatic disease. We refer to them as long survivors. All the patients referring to our Department are asked to give a written informed consent for conservation of biological material that could be use for research propose.

In the study reported herein, we selected 4 patients with metastatic gastric cancer, treated with the same therapeutic regimen, who have not reached clinical remission or have presented early disease relapse. We refer to them as normal survivors. Clinical characteristics of patients are reported in Table I. Long survivors present a median disease-free and overall survival of 75 months (66-82 months), while normal responders die within one year (median 13 months; 9-33 months).

\section{Expression profiling}

Custom Taqman Low Density Array construction. Bioinformatic procedure for the design of the database of genes to be evaluated was based on medical literature reporting studies already performed in gastric cancer evaluating polymorphisms and expression profiles of a selection of genes related to the response to CT and survival. This was done through GeneCards database (www.genecards.org), integrated with a literature mining approach both software assisted (MedMiner) (5) and manually edited. Ninety-three genes were selected and used to create the Custom Taqman Low Density array (TLDA, Applied Biosystem) (Table II and Fig. 1).

RNA extraction. RNA was extracted from paraffin-embedded slices, using Recover All Total Nucleic Acid Isolation Kit (Ambion), following manufacturer's instructions. For each patient, three different samples of the biopsy were identified by the pathologist as proper cancer tissues and RNA was extracted independently for each sample, in order to be more representative of the tumoral tissue.

Quantitative real-time $R T-P C R$ on TLDA. Custom TLDA containing a set of 93 cancer-related genes (and 3 endogenous control genes: $\beta_{2}$ M-Hs99999907_m1, GAPDHHs99999905_m1, GUSB-Hs99999908_m1) were used for expression studies. cDNAs for real-time RT-PCR were produced using the High Capacity cDNA Archive kit (Applied Biosystem) following the manufacturer's protocol. Inventoried assays were chosen up to $85 \mathrm{bp}$, of _m type, being assay designed at the exon-exon boundaries of the genes. Amplifications on the TLDA were done on a 7900HT instrument (Applied Biosystem). Forty-five cycles of PCR were performed. Each cDNA was tested in three independent PCR reactions.

Gene expression data analysis. Quantitative real-time RTPCR data were extracted using SDS 2.2.2 software (Applied Biosystem). Microsoft Excel file containing SDS results was acquired and analyzed in R v2.10.1 software environment. 
Table II. List of 93 cancer-related genes investigated on Taqman Low Density Array.

\begin{tabular}{|c|c|c|}
\hline Gene symbol & Gene description & Assay no. (AB) \\
\hline $\mathrm{ABCB} 1$ & ATP-binding cassette, sub-family B (MDR/TAP), member 1 & Hs00184500_m1 \\
\hline $\mathrm{ABCC} 1$ & ATP-binding cassette, sub-family C (CFTR/MRP), member 1 & Hs00219905_m1 \\
\hline $\mathrm{ABCC} 2$ & ATP-binding cassette, sub-family C (CFTR/MRP), member 2 & Hs00166123_m1 \\
\hline $\mathrm{ABCC} 3$ & ATP-binding cassette, sub-family C (CFTR/MRP), member 3 & Hs00358656_m1 \\
\hline $\mathrm{ABCC} 5$ & ATP-binding cassette, sub-family C (CFTR/MRP), member 5 & Hs00194701_m1 \\
\hline ABCG2 & ATP-binding cassette, sub-family G (WHITE), member 2 & Hs00184979_m1 \\
\hline AKT1 & v-akt murine thymoma viral oncogene homolog 1 & Hs00178289_m1 \\
\hline APEX1 & APEX nuclease (multifunctional DNA repair enzyme) 1 & Hs00172396_m1 \\
\hline ATM & ataxia telangiectasia mutated & Hs00175892_m1 \\
\hline BAX & BCL2-associated X protein & Hs00180269_m1 \\
\hline BCL2 & B-cell CLL/lymphoma 2 & Hs00608023_m1 \\
\hline BCL2L1 & BCL2-like 1 & Hs00169141_m1 \\
\hline BIRC4 & baculoviral IAP repeat-containing 4 & Hs00236913_m1 \\
\hline CALD1 & caldesmon 1 & Hs00189021_m1 \\
\hline CASP3 & caspase 3 , apoptosis-related cysteine peptidase & Hs00234385_m1 \\
\hline CASP9 & caspase 9 , apoptosis-related cysteine peptidase & Hs00154261_m1 \\
\hline CCNB1 & cyclin B1 & Hs00259126_m1 \\
\hline CCND1 & cyclin D1 & Hs00277039_m1 \\
\hline CDH1 & cadherin 1 , type 1, E-cadherin (epithelial) & Hs00170423_m1 \\
\hline CDKN1A & cyclin-dependent kinase inhibitor 1A (p21, Cip1) & Hs00355782_m1 \\
\hline CDKN1B & cyclin-dependent kinase inhibitor 1B (p27, Kip1) & Hs00153277_m1 \\
\hline CHFR & checkpoint with forkhead and ring finger domains & Hs00217191_m1 \\
\hline CYP1A1 & cytochrome $\mathrm{P} 450$, family 1 , subfamily A, polypeptide 1 & Hs00153120_m1 \\
\hline CYP2C8 & cytochrome $\mathrm{P} 450$, family 2 , subfamily $\mathrm{C}$, polypeptide 8 & Hs00946140_g1 \\
\hline CYP3A4 & cytochrome P450, family 3 , subfamily A, polypeptide 4 & Hs00430021_m1 \\
\hline DDIT3 & DNA-damage-inducible transcript 3 & Hs00358796_g1 \\
\hline DHFR & dihydrofolate reductase & Hs00758822_s1 \\
\hline DPYD & dihydropyrimidine dehydrogenase & Hs00559279_m1 \\
\hline $\mathrm{E} 2 \mathrm{~F} 1$ & E2F transcription factor 1 & Hs00153451_m1 \\
\hline ECGF1 & endothelial cell growth factor 1 (platelet-derived) & Hs00157317_m1 \\
\hline EGF & epidermal growth factor (beta-urogastrone) & Hs00153181_m1 \\
\hline EGFR & $\begin{array}{l}\text { epidermal growth factor receptor (erythroblastic leukemia } \\
\text { viral (v-erb-b) oncogene homolog, avian) }\end{array}$ & Hs00193306_m1 \\
\hline ERBB2 & $\begin{array}{l}\text { v-erb-b2 erythroblastic leukemia viral oncogene homolog } 2 \text {, } \\
\text { neuro/glioblastoma derived oncogene homolog (avian) }\end{array}$ & Hs00170433_m1 \\
\hline ERBB3 & v-erb-b2 erythroblastic leukemia viral oncogene homolog 3 (avian) & Hs00176538_m1 \\
\hline ERBB4 & v-erb-a erythroblastic leukemia viral oncogene homolog 4 (avian) & Hs00171783_m1 \\
\hline ERCC1 & $\begin{array}{l}\text { excision repair cross-complementing rodent repair deficiency, } \\
\text { complementation group } 1\end{array}$ & Hs00157415_m1 \\
\hline ERCC2 & $\begin{array}{l}\text { excision repair cross-complementing rodent repair deficiency, } \\
\text { complementation group } 2 \text { (xeroderma pigmentosum D) }\end{array}$ & Hs00361161_m1 \\
\hline FGFR4 & fibroblast growth factor receptor 4 & Hs00242558_m1 \\
\hline GADD45A & growth arrest and DNA-damage-inducible, alpha & Hs00169255_m1 \\
\hline GSTP1 & glutathione S-transferase pi & Hs00168310_m1 \\
\hline GSTT1 & glutathione S-transferase theta 1 & Hs00184475_m1 \\
\hline HGF & hepatocyte growth factor (hepapoietin A; scatter factor) & Hs00300159_m1 \\
\hline HIF1A & hypoxia-inducible factor 1 , alpha subunit (basic helix-loop-helix & Hs00153153_m1 \\
\hline
\end{tabular}


Table II. Continued.

\begin{tabular}{|c|c|c|}
\hline Gene symbol & Gene description & Assay no. (AB) \\
\hline HPRT1 & hypoxanthine phosphoribosyltransferase 1 (Lesch-Nyhan syndrome) & Hs99999909_m1 \\
\hline HRAS & v-Ha-ras Harvey rat sarcoma viral oncogene homolog & Hs00610483_m1 \\
\hline JUN & jun oncogene & Hs99999141_s1 \\
\hline KDR & kinase insert domain receptor (a type III receptor tyrosine kinase) & Hs00176676_m1 \\
\hline KIT & v-kit Hardy-Zuckerman 4 feline sarcoma viral oncogene homolog & Hs00174029_m1 \\
\hline KRAS & v-Ki-ras2 Kirsten rat sarcoma viral oncogene homolog & Hs00364282_m1 \\
\hline MAPK1 & mitogen-activated protein kinase 1 & Hs00177066_m1 \\
\hline MAPK3 & mitogen-activated protein kinase 3 & Hs00385075_m1 \\
\hline MAPK8 & mitogen-activated protein kinase 8 & Hs00177083_m1 \\
\hline MDK & midkine (neurite growth-promoting factor 2) & Hs00171064_m1 \\
\hline MET & met proto-oncogene (hepatocyte growth factor receptor) & Hs00179845_m1 \\
\hline MGMT & O-6-methylguanine-DNA methyltransferase & Hs00172470_m1 \\
\hline MMP1 & matrix metallopeptidase 1 (interstitial collagenase) & Hs00899658_m1 \\
\hline MMP3 & matrix metallopeptidase 3 (stromelysin 1, progelatinase) & Hs00968308_m1 \\
\hline MRE11A & MRE11 meiotic recombination 11 homolog A (S. cerevisiae) & Hs00271551_m1 \\
\hline MTHFR & 5,10-methylenetetrahydrofolate reductase (NADPH) & Hs00195560_m1 \\
\hline MUC1 & mucin 1 , cell surface associated & Hs00159357_m1 \\
\hline MVP & major vault protein & Hs00245438_m1 \\
\hline OGG1 & 8-oxoguanine DNA glycosylase & Hs00213454_m1 \\
\hline PDGFRA & platelet-derived growth factor receptor, alpha polypeptide & Hs00998026_m1 \\
\hline PIK3CA & phosphoinositide-3-kinase, catalytic, alpha polypeptide & Hs00180679_m1 \\
\hline PLK1 & polo-like kinase 1 (Drosophila) & Hs00153444_m1 \\
\hline POR & P450 (cytochrome) oxidoreductase & Hs00287016_m1 \\
\hline PRNP & $\begin{array}{l}\text { prion protein (p27-30) (Creutzfeldt-Jakob disease, Gerstmann- } \\
\text { Strausler-Scheinker syndrome, fatal familial insomnia) }\end{array}$ & Hs00175591_m1 \\
\hline PTEN & phosphatase and tensin homolog (mutated in multiple advanced cancers 1) & Hs01920652_s1 \\
\hline PTGS2 & $\begin{array}{l}\text { prostaglandin-endoperoxide synthase } 2 \text { (prostaglandin } \mathrm{G} / \mathrm{H} \text { synthase and } \\
\text { cyclooxygenase) }\end{array}$ & Hs00153133_m1 \\
\hline RAD50 & RAD50 homolog (S. cerevisiae) & Hs00194871_m1 \\
\hline REG4 & regenerating islet-derived family, member 4 & Hs00230746_m1 \\
\hline RRM1 & ribonucleotide reductase $\mathrm{M} 1$ polypeptide & Hs00168784_m1 \\
\hline SMAD4 & SMAD family member 4 & Hs00232068_m1 \\
\hline SOD2 & superoxide dismutase 2 , mitochondrial & Hs00167309_m1 \\
\hline $\mathrm{SRC}$ & v-src sarcoma (Schmidt-Ruppin A-2) viral oncogene homolog (avian) & Hs00178494_m1 \\
\hline TGFA & transforming growth factor, alpha & Hs00608187_m1 \\
\hline TGFB1 & transforming growth factor, beta 1 & Hs00171257_m1 \\
\hline TNF & tumor necrosis factor (TNF superfamily, member 2) & Hs00174128_m1 \\
\hline TOP1 & topoisomerase (DNA) I & Hs00243257_m1 \\
\hline TOP2A & topoisomerase (DNA) II alpha $170 \mathrm{kDa}$ & Hs00172214_m1 \\
\hline TOP2B & topoisomerase (DNA) II beta $180 \mathrm{kDa}$ & Hs00172259_m1 \\
\hline TP53 & tumor protein p53 (Li-Fraumeni syndrome) & Hs00153349_m1 \\
\hline TP63 & tumor protein p63 & Hs00186613_m1 \\
\hline TP73 & tumor protein $\mathrm{p} 73$ & Hs00232088_m1 \\
\hline TSG101 & tumor susceptibility gene 101 & Hs00173072_m1 \\
\hline TUBB & tubulin, beta & Hs00742828_s1 \\
\hline TYMS & thymidylate synthetase & Hs00426591_m1 \\
\hline UGT1A1 & UDP glucuronosyltransferase 1 family, polypeptide A1 & Hs00153559_m1 \\
\hline UGT1A10 & UDP glucuronosyltransferase 1 family, polypeptide A10 & \\
\hline
\end{tabular}


Table II. Continued.

Gene symbol

Gene description

Assay no. (AB)

UGT1A3

UGT1A4

UGT1A5

UGT1A6

UGT1A7

UGT1A8

UGT1A9

UMPS

VEGFA

$\mathrm{XPC}$

XRCC1

XRCC3
UDP glucuronosyltransferase 1 family, polypeptide A3

UDP glucuronosyltransferase 1 family, polypeptide A4

UDP glucuronosyltransferase 1 family, polypeptide A5

UDP glucuronosyltransferase 1 family, polypeptide A6

UDP glucuronosyltransferase 1 family, polypeptide A7

UDP glucuronosyltransferase 1 family, polypeptide A8

UDP glucuronosyltransferase 1 family, polypeptide A9

uridine monophosphate synthetase (orotate phosphoribosyl

Hs00923517_m1

transferase and orotidine-5'-decarboxylase)

vascular endothelial growth factor A

Hs00900054_m1

Hs00190295_m1

Hs00959834_m1

Hs00193725_m1
In order to investigate the expression level of the 93 cancer-related genes, an analysis of $\mathrm{Ct}$ determined (CDA) and an analysis of $\mathrm{Ct}$ undetermined (CUA) were performed. In CDA the data transformations were applied in the following order: i) pre-processed for quality assessment. To perform this, genes with $<3$ out of ten replicates and $\mathrm{Ct}$ undetermined results were removed. For each cDNA, the values of the endogenous control $\beta_{2} \mathrm{M}$ more than 70th percentile were not considered in the subsequent analysis. For each patient, the $\mathrm{Ct}$ values were obtained as mean of the replicates of each gene; genes with $>30 \%$ missing values were filtered out. ii) Data were normalized using the delta method (Applied Biosystem) towards the endogenous control gene $\beta_{2} \mathrm{M}$. iii) Differential gene expression analysis was carried out in the two groups of cases and controls using the t-test.

The genes, which were removed from the CDA after being pre-processed for quality assessment, were considered in the CUA. In particular, the frequency of $\mathrm{Ct}$ undetermined was tested between cases and controls throughout the Fisher's exact test. As to the genes resulting statistically significant, the $\mathrm{Ct}$ undetermined was set to 45 (as the number of PCR cycles performed) and the differential gene expression analysis using the t-test was performed again.

In all analyses, the tests were adjusted for multiple comparisons using the false discovery rate (FDR) method (6) and genes with an estimated FDR $<0.05$ were selected as significant. Similarities among groups of patients and among groups of genes were studied using hierarchical clustering analysis (Ward method, Euclidean distance) on significant genes in each study comparison.

Genotyping of SNPS

DNA extraction. DNA was extracted from leukocytes of peripheral blood using EZ1 DNA blood kit (Qiagen) and from paraffin-embedded slices using standard xylene-phenol protocol. In order to empower statistical analysis, DNA was extracted from peripheral blood of additional control groups: i) 10 normal survivors affected by gastric cancer; ii) 115 patients affected by various cancers; iii) 232 patients affected by breast and head-neck cancer.

Pyrosequencing. Single nucleotide polymorphism (SNP) analysis was done through pyrosequencing using the chemotherapy response kits (Diatech). The following SNPs were characterized: MTHFR-C677T, MTHFR-A1298C, DPYD IVS14+1G>A, the 5' UTR region of TYMS (TSER), the 6-bp deletion at nucleotide 1494 within the 3'UTR of TYMS (+6 bp/ -6 bp 3'UTR), Ile105Val in GSTP1, C8092A (rs3212986) and T19007C (rs11615) in ERCC1, G28152A in XRCC1 (Arg399Gln), C1236T and C3435T in ABCB1, CYP3A4*1B $-392 \mathrm{~A}>\mathrm{G}$ and CYP3A5*3 22893G $>\mathrm{A}, \mathrm{ABCC} 2-24 \mathrm{C}>\mathrm{T}$.

Direct sequencing and restriction enzyme analysis was used for the G/C SNP at the 12th nucleotide in the second repeat of the $3 R$ allele $(3 R G>3 R C)$. A custom assay for pyrosequencing was designed for the genotyping of $\mathrm{p} 53$ codon 72 polymorphism (forward and sequencing primer: 5'caacgttctggtaaggacaagg-3'; reverse primer: 5'-ccggtgtaggag ctgctgg-3').

SNPs statistical analysis. In SNP analysis long survivors were compared with the 3 different sets of controls above reported. When the frequency distribution of a polymorphism was known both in cases and controls, the association between the long survival and the polymorphic variants of selected genes was tested throughout the Fisher's exact test. A binomial exact test was performed to compare the genomic profile of long survivors with data from literature.

\section{Results}

Expression profiles. The expression study was performed in the three long survivors and the four normal survivors on the TLDA platform of 93 cancer-related genes (Table II and Fig. 1). The inclusion criteria of pre-processed for quality 


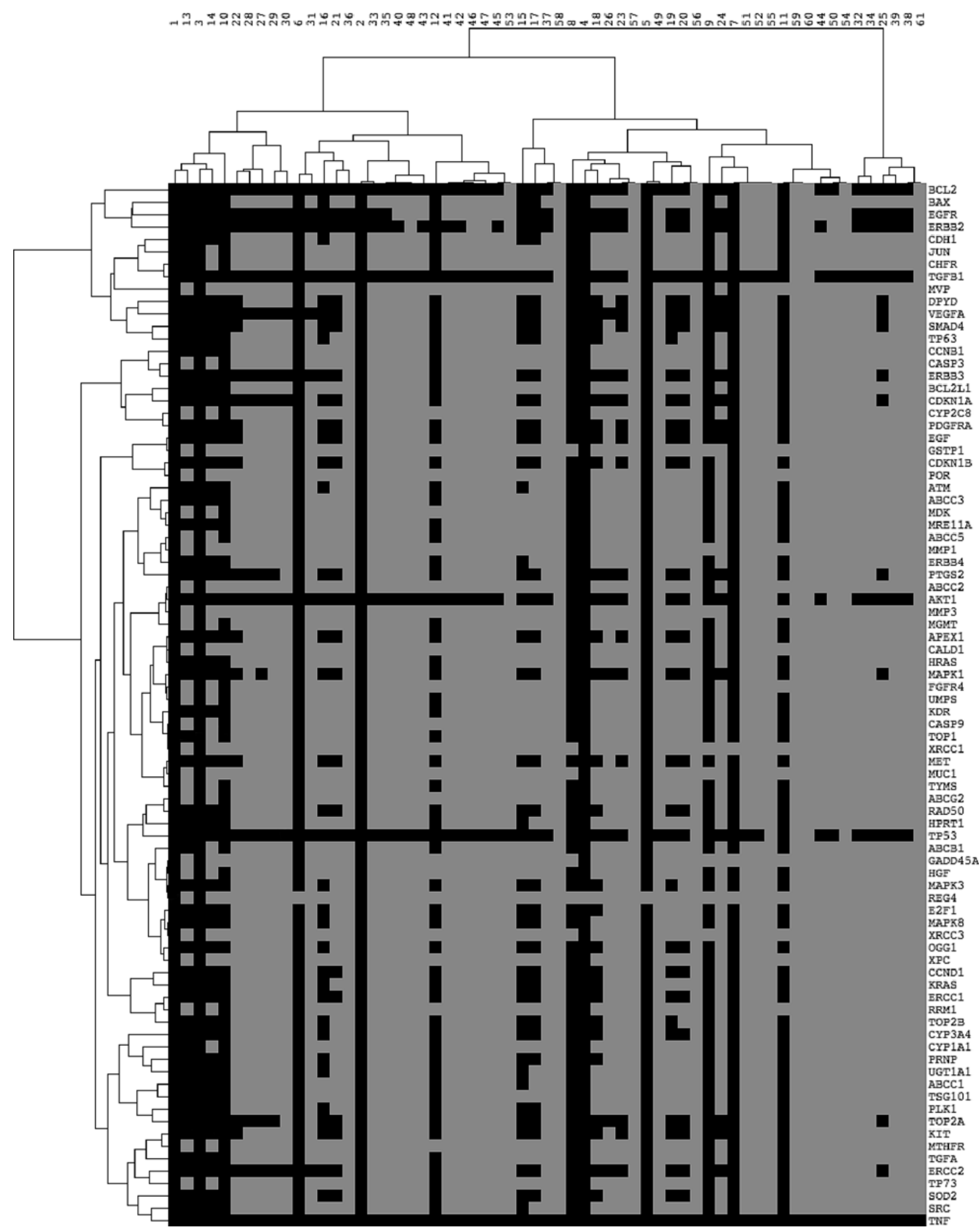

Figure 1. Clustering of selected genes according to Gene Ontology (GO) classification (http://www.geneontology.org/). The genes are classified according to GO categories (biological process). Hierarchical clustering was then performed owing to the hierachical structure GO entries for grouping together genes involved in similar biological pathways.

assessment (see Gene expression data analysis paragraph) were not met by five genes (i.e., CYP1A1, CYP3A4, ERBB4, EGF and TP63). Those genes were removed from the CDA and were considered in the CUA.
Expression analysis in long survivors revealed a significant upregulation of PTEN. Moreover, a consistent upregulation of GADD45a, MAPK1, TP63 genes and a consistent downregulation of the EGF gene, which presented 


\begin{tabular}{|c|c|c|c|c|c|c|}
\hline Genes & $\Delta C t$ cases & $\Delta C t$ controls & $-\Delta \Delta C t$ & P.value & P.value.adjust & \\
\hline PTEN-Hs01920652_s1 & 8.0705 & 13.1724 & 5.1019 & 0.0005 & 0.0430 & *t \\
\hline GADD45A-Hs00169255_m1 & 10.0543 & 14.1033 & 4.0491 & 0.0053 & 0.2182 & *t \\
\hline MAPK1-Hs00177066_m1 & 7.5945 & 10.2277 & 2.6332 & 0.0073 & 0.2182 & $* \star$ \\
\hline EGF-Hs00153181_m1 & 21.1744 & 14.2098 & -6.9647 & 0.0120 & 0.0600 & * \\
\hline TP63-Hs00186613_m1 & -6.6869 & 18.2299 & 24.9168 & 0.0350 & 0.0875 & * \\
\hline CYP3A4-Hs00430021_m1 & 16.9003 & 21.1641 & 4.2637 & 0.1191 & 0.1824 & * \\
\hline CYP1A1-Hs00153120_m1 & 16.8978 & 21.1641 & 4.2663 & 0.1459 & 0.1824 & * \\
\hline ERBB 4-Hs00171783_m1 & 16.8254 & 20.7188 & 3.8934 & 0.1998 & 0.1998 & * \\
\hline
\end{tabular}
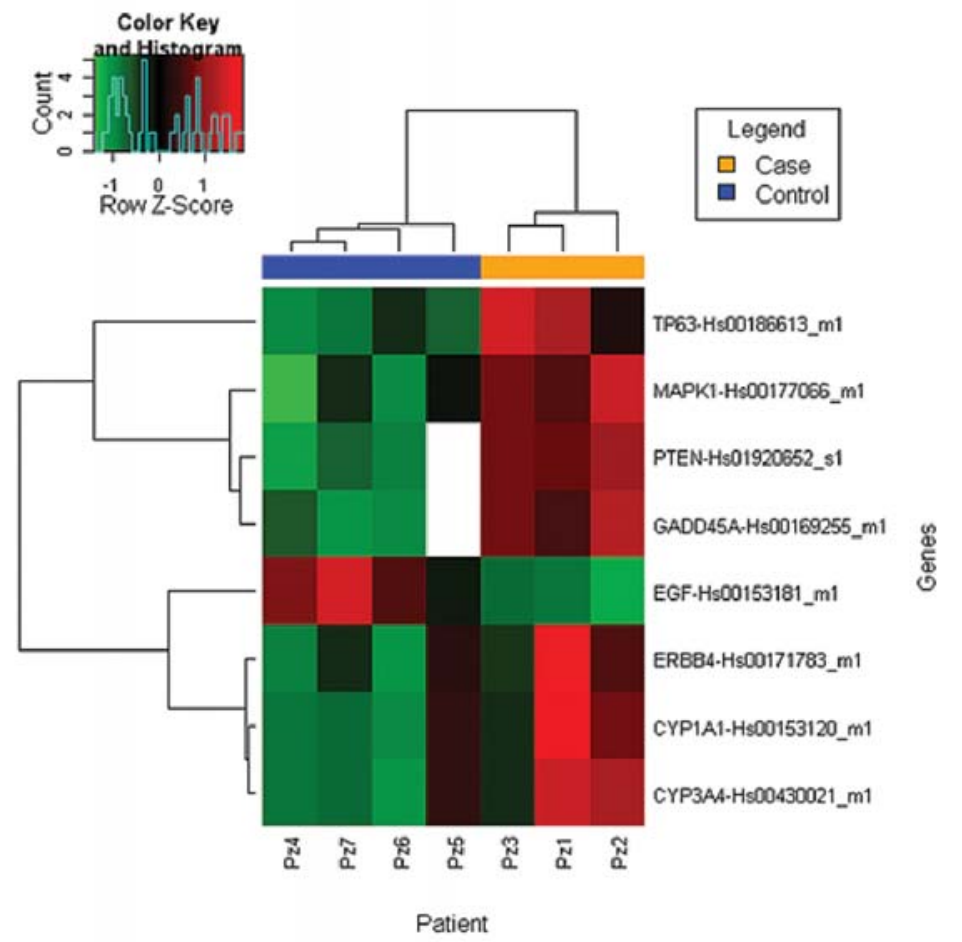

Figure 2. Expression analysis in long survivors (cases) versus normal survivors (controls). $\Delta \mathrm{Ct}$ cases, mean Delta $\mathrm{Ct}$ cases; $\Delta \mathrm{Ct}$ controls, mean Delta Ct controls; - $\Delta \Delta \mathrm{Ct}$, Delta Delta Ct, P-value, p-value of the t-test; P-value.adjust, p-value adjusted; ${ }^{* *}$ results from CDA (Ct determined) analysis; ${ }^{*}$ results from CUA (Ct undetermined) analysis.

a p $<0.05$, were found (Fig. 2). As regards CYP1A1, CYP3A4 and ERBB4 genes, the Fisher's exact test showed a higher frequency of $\mathrm{Ct}$ undetermined in controls, revealing that those genes were upregulated in long survivors. Having re-done the t-test analysis by replacing the $\mathrm{Ct}$ undetermined data with $\mathrm{Ct}=45$, differential expression was seen for CYP1A1, CYP3A4 and ERBB4 genes (Fig. 2), although not reaching statistical significance.

Genetic screening. In addition to genes involved in 5-FU (TYMS, DYPD, MTHFR) and cisplatin (GSTP, ERCC1, XRCC1) metabolism, we studied selected polymorphisms in CYP3A4, CYP3A5, ABCC2, ABCB1 genes, in order to explain, at least partially, the expression data we observed in the tissues analysed. Moreover, we analysed p53 codon 72 SNP in our cohort of patients and in additional 232 blood samples from cancer patients.

Genetic screening of SNPs did not reveal interesting genotypes correlated with the long survivor phenotype, except ERCC1 C8092A (Table III). ERCC1 C8092A was associated with survival in patients with advanced gastric cancer treated with standard chemotherapy ( $\mathrm{p}$-value versus 4 controls $=0.038$; versus 10 controls $=0.035$; versus 115 controls $=0.027$ ).

All long survivors presented the G/G genotype at codon 72 of the p53 gene, related to a better response rate to $\mathrm{CT}$, in respect to the 4 normal survivors, where the frequency was $40 \%$ and to our control population of 232 tumor samples, where frequency was $50 \%$ (data not shown). Although the data did not reach statistical significance.

\section{Discussion}

We present a genetic and gene expression analysis of three gastric patients, metastatic at presentation, who showed unexpected long-term survival, still continuing. These cases were compared to similar patients showing a standard behaviour. Our results revealed a common signature among 


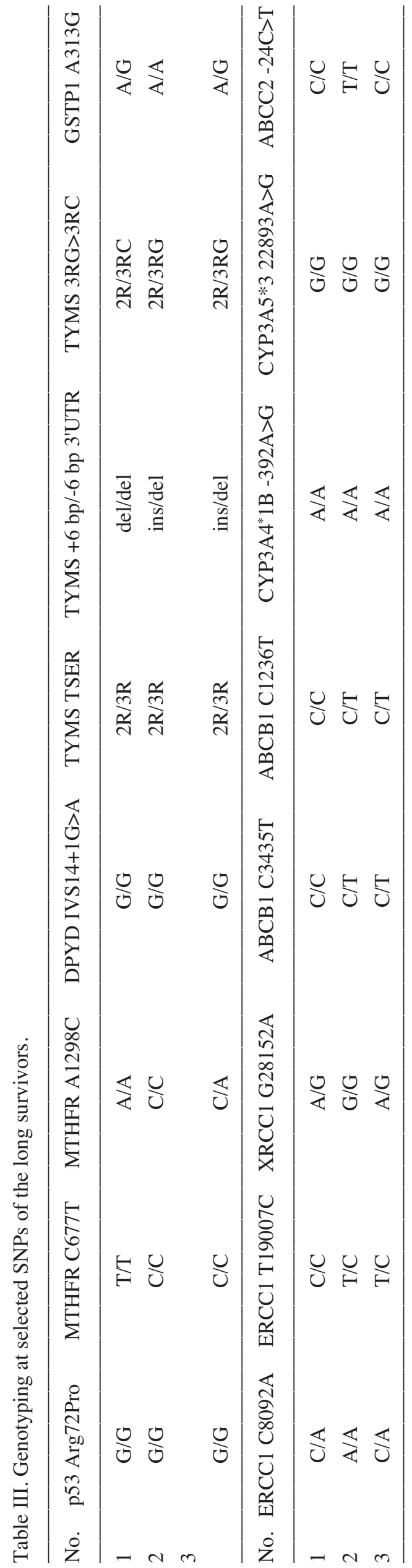

the long survivors that distinguish them from the control cases. The 3 patients considered are still alive and disease-free at 66-82 months from chemotherapy. Data from the literature suggest that expected 5-year survival at stage IV is of $<5 \%$ (7). Indeed, the 4 patients used as control group survived 9-33 months. All the 7 patients received epirubicin/cisplatin/5-FU (ECF) regimen and the control group had even a second line CT.

To evaluate the possibility of using molecular profiling as predictor of clinical outcome and survival in metastatic gastric cancer, we used quantitative RT-PCR to measure the expression of 93 genes on a Custom Taqman Low Density Array, designed by literature mining using MedMiner for data retrieval of cancer-treatment-prolonged-survival-related genes. Selected genes are involved in drug metabolism and resistance, DNA repair, cell cycle regulation and growth factors signalling.

Recently, pharmacogenetics and large-scale molecular techniques such as DNA microarrays have contributed to our understanding of the molecular complexity of gastric cancer. Prognostic classification according to gene expression profile has been achieved $(4,8)$ and many candidate genes for the prediction of patient survival have been reported in gastric cancer $(9,10)$. Expression analysis in our long survivors revealed a significant upregulation of PTEN and a consistent upregulation of GADD45a, MAPK1 and TP63 genes.

GADD45a is involved in cell cycle control and stimulates DNA repair. Napieralski et al (11) studied pre-therapeutic paraffin-embedded biopsies of 61 advanced gastric cancer patients, who received a 5-FU-cisplatin-based CT. They analysed the 5-FU-related genes TYMS, DPYD and TP and the cisplatin-related genes ERCC1, ERCC4, KU80 and GADD45a by quantitative real-time PCR. GADD45a and TP levels showed weak associations with response, but GADD45a expression correlated with survival.

There is ample evidence that the functions of GADD45 proteins are mediated via interactions with other cellular proteins implicated in cell cycle regulation and the response of cells to extrinsic stress, including p21, Cdc2/cyclin B1 and p38/JNK.

Mitogen-activated protein kinase 1 (MAPK1) also known as p38, ERK or ERK2, is a member of the MAP kinase family, involved in a wide variety of cellular processes such as proliferation, differentiation, transcription regulation and development. Its activity is determined by the upstream Ras/Raf/MEK cascade and the deactivating MAPK phosphatases 1, 2, and 3. Wu et al (12) showed that ERK1/2 phosphorylation and c-Jun expression were significantly lowered in gastric cancer compared with the non-cancer adjacent tissues.

The phosphatase and tensin homolog mutated on chromosome ten (PTEN) gene product is a protein tyrosine phosphatase that participates in modulating the phosphoinositide 3-kinase pathway which antagonizes protein tyrosine kinases. Several authors investigated the correlation between clinicopathologic variables, including survival, and the loss of PTEN expression in gastric adenocarcinoma patients. Altered PTEN expression was significantly associated with tumor depth and size, lymphatic invasion, advanced stage, pTNM stage, and patient survival (13-15). 
TP63 gene is a p53 homologue that encodes proteins with transactivation, DNA-binding and tetramerization domains. No clear published data on TP63 expression in gastric cancer exist. A few reports are related to other malignancies. Several authors found that decreased expression of TP63 and decreased p63 immunoreactivity is significantly associated with advanced tumor stage and grade in renal malignancies (16) and in gallbladder cancer (17). Hallack et al (18) found that p63 was expressed in more than $50 \%$ of malignant cells in diffuse large B-cell lymphomas and p63(+) patients had better disease-free survival than those who were negative.

In addition, in the three long survivors we found also an upregulation of ERBB4, CYP1A1 and CYP3A4 genes. Although these genes are not differentially expressed according to the t-test analysis, results supplied by Fisher's exact test seem sufficient to take them into consideration.

HER 1 and HER2 overexpression in gastric cancers are thought to be prognostic factors and targets of novel biological agents. Hayashi and collaborators (19) found that HER3 overexpression was associated with a significantly worse survival and was an independent prognostic factor in gastric cancer, whereas HER1, HER2 or HER4 overexpression did not show any such association. The effect of HER4 expression in gastric cancer has not been fully elucidated. Published data on HER4 in breast cancer reported conflicting results $(20,21)$. However, Sassen et al (22) demonstrated that HER4 expression prolongs overall survival in Herceptin-treated breast cancer patients. HER4 expression was not associated with the prognosis of patients with colorectal cancer, although its membranous expression was associated with involved lymph nodes.

Because CYP3As inactivate many anticancer drugs, an overexpression of CYP3As in tumors could result in an increased intratumoral drug inactivation and decreased drug efficacy. Rodríguez-Antona et al (23) found that a high tumoral expression of CYP3A4 was significantly associated with a lower complete remission rate in peripheral $\mathrm{T}$-cell lymphomas (PTCL). They concluded that a high CYP3A4 tumoral expression could be useful to predict poor response to the standard PTCL chemotherapy, but it does not affect survival. However, CYP3A4 and CYP3A1 are not the main enzymes involved in the metabolism of the drugs of the ECF regimen used in these patients. Epidermal growth factor (EGF) promotes the growth of cells of both ectodermal and mesodermal origin, and plays an important role in cellular proliferation and differentiation. Studies investigating EGF receptor in gastric cancer patients have shown that an increased level was associated with poor prognosis (24). Gastric cancer patients with EGFR expression and low ligand levels had better outcomes with cetuximab/mFOLFOX6 treatment (25). In accordance with those studies, we found that EGF is down-regulated in our cohort of long survivals.

Since genetic polymorphisms could explain variations in pharmacokinetics, in activity and in expression of the target or of the proteins involved in the mechanism of action of the drug, we analyzed functional genetic variations in metabolizing enzymes of 5-FU (TYMS, DPYD, MTHFR) and of cisplatin (GSTP, ERCC1, XRCC1). We added drug resistance $\mathrm{ABCB} 1$ and $\mathrm{ABCC} 2$ genes and $\mathrm{CYP} 3 \mathrm{~A} 4$, that we have found differentially expressed in long survivors versus normal survivors.
Moreover, several studies have already suggested that the p53 codon 72 polymorphism modulates the p53-dependent apoptotic capacity. In particular, the Arg/Arg genotype correlates with better response rate to $\mathrm{CT}$ and longer time to progression in patients with advanced gastric cancer (26). Huang and co-authors (27) found same association in gastric cancer patients who benefit from oxaliplatin-based adjuvant CT .

We did not find any statistical difference at this locus between long and normal survivors, although all the long survivors presented the G/G genotype. This lack of significance may be related, of course, to the low number of long survivors.

Genetic screening did not reveal any other interesting genotype correlated with the long survival, a part from SNP C8092A in ERCC1 (excision repair cross complementing 1). Among the members of the nucleotide excision repair system (NER) family, the product of the ERCC1 gene is necessary for the repair of the damaged DNA due by cross-link interchain and intrachain induced by cis-platinum and its derivates. The expression of ERCC1 gene has been correlated with the clinical outcome of non-small cell lung cancer (NSCLC) and colon cancer treated with cisplatinum and oxaliplatinum, in particular increased responsiveness and prolonged survival has been demonstrated in situations of reduced expression of the ERCC1 gene (28).

C8092A (rs3212986) and T19007C (rs11615) are two common polymorphisms of ERCC1, that have been demonstrated to impact clinical outcome of patients receiving platinum-based chemotherapy. In particular, Okuda et al (29) studied C8092A polymorphism in advanced NSCLC patients treated with platinum-based chemotherapy and found that $\mathrm{C} / \mathrm{C}$ genotype at codon 8092 was associated with better prognosis than $\mathrm{C} / \mathrm{A}$ or $\mathrm{A} / \mathrm{A}$ and the wild-type $\mathrm{C} / \mathrm{C}$ of the codon 118 was associated with better prognosis than $\mathrm{C} / \mathrm{T}$ or $\mathrm{T} / \mathrm{T}$ types. On the contrary, Kalikaki et al (30) found significant association between the ERCC1 C8092A polymorphism and overall survival in advanced NSCLC, suggesting that any copies of the A allele were associated with an improved outcome.

In conclusion, the present study has shed light on explaining long survival in our metastatic gastric cancer patients. We have focused on a set of markers targeting specific pathways or a set of pathways that may be involved in cellular response to cytotoxic agents. However, it is clear that the cancer phenotype is a sum total of genetic and epigenetic alterations and, therefore, the ultimate response to cytotoxic therapy and survival in cancer is also likely to be dictated by these genetic and epigenetic changes involving perhaps several thousands of genes within the cancer genome.

\section{Acknowledgments}

The authors thank Dr Cristina Zanchi from Applied Biosystem for technical assistance with microfluid card experiments, Dr Vita Mutri and Carmine Pinto (Department of Medical Oncology, S. Orsola-Malpighi Hospital, Bologna, Italy) for their help in collecting biological samples and general discussion and Mr. Domenico Carratta for technical help in preparing manuscript figures. This work was partially supported by Fondazione Cassa Risparmio Torino. 


\section{References}

1. Devesa SS, Blot WJ and Fraumeni JF Jr: Changing patterns in the incidence of esophageal and gastric carcinoma in the United States. Cancer 83: 2049-2053, 1998.

2. Wolschke C, Goekkurt E, A-Batran SE and Hossfeld DK: Pharmacogenetics of extraordinary responses to 5-FU/cisplatin chemotherapy in advanced gastric cancer - report of 2 cases. Onkologie 28: 589-592, 2005.

3. Ichikawa W: Prediction of clinical outcome of fluoropyrimidinebased chemotherapy for gastric cancer patients, in terms of the 5-fluorouracil metabolic pathway. Gastric Cancer 9: 145-155, 2006.

4. Fareed KR, Kaye P, Soomro IN, et al: Biomarkers of response to therapy in oesophago-gastric cancer. Gut 58: 127-143, 2009.

5. Tanabe L, Scherf U, Smith LH, Lee JK, Hunter L and Weinstein JN: MedMiner: an Internet text-mining tool for biomedical information, with application to gene expression profiling. Biotechniques 27: 1210-1214, 1216-1217, 1999.

6. Benjamini Y and Hochberg Y: Controlling the false discovery rate: a practical and powerful approach to multiple testing. J Roy Statist Soc Ser B 57: 289-300, 1995.

7. Janunger KG, Hafström L, Nygren P and Glimelius B: SBUgroup: Swedish Council of Technology Assessment in Health Care. A systematic overview of chemotherapy effects in gastric cancer. Acta Oncol 40: 309-326, 2001.

8. Lee HS, Cho SB, Lee HE, et al: Protein expression profiling and molecular classification of gastric cancer by the tissue array method. Clin Cancer Res 13: 4154-4163, 2007.

9. Marchet A, Mocellin S, Belluco C, et al: Gene expression profile of primary gastric cancer: towards the prediction of lymph node status. Ann Surg Oncol 14: 1058-1064, 2007.

10. Xu ZY, Chen JS and Shu YQ: Gene expression profile towards the prediction of patient survival of gastric cancer. Biomed Pharmacother 64: 133-139, 2010.

11. Napieralski R, Ott K, Kremer M, et al: Combined GADD45A and thymidine phosphorylase expression levels predict response and survival of neoadjuvant-treated gastric cancer patients. Clin Cancer Res 11: 3025-3031, 2005.

12. Wu WK, Sung JJ, Yu L, Li ZJ, Chu KM and Cho CH: Constitutive hypophosphorylation of extracellular signal-regulated kinases-1/2 and down-regulation of c-Jun in human gastric adenocarcinoma. Biochem Biophys Res Commun 373: 330-334, 2008.

13. Tamura G: Alterations of tumor suppressor and tumor-related genes in the development and progression of gastric cancer. World J Gastroenterol 12: 192-198, 2006.

14. Bai Z, Ye Y, Chen D, et al: Homeoprotein Cdx2 and nuclear PTEN expression profiles are related to gastric cancer prognosis. APMIS 115: 1383-1390, 2007.

15. Zheng H, Takahashi H, Murai Y, et al: Low expression of FHIT and PTEN correlates with malignancy of gastric carcinomas: tissue-array findings. Appl Immunohistochem Mol Morphol 15: 432-440, 2007

16. Tuna B, Unlu M, Aslan G, Secil M and Yorukoglu K: Diagnostic and prognostic impact of p63 immunoreactivity in renal malignancies. Anal Quant Cytol Histol 31: 118-122, 2009.
17. Urist MJ, Di Como CJ, Lu ML, et al: Loss of p63 expression is associated with tumor progression in bladder cancer. Am J Pathol 161: 1199-1206, 2002.

18. Hallack Neto AE, Siqueira SA, Dulley FL, Ruiz MA, Chamone DA and Pereira J: p63 protein expression in high risk diffuse large B-cell lymphoma. J Clin Pathol 62: 77-79, 2009.

19. Hayashi M, Inokuchi M, Takagi Y, et al: High expression of HER3 is associated with a decreased survival in gastric cancer. Clin Cancer Res 14: 7843-7849, 2008.

20. Su HI, Sammel MD, Velders L, et al: Association of cyclophosphamide drug-metabolizing enzyme polymorphisms and chemotherapy-related ovarian failure in breast cancer survivors. Fertil Steril (In press).

21. Aubele M, Auer G, Walch AK, et al: PTK (protein tyrosine kinase)-6 and HER2 and 4, but not HER1 and 3 predict longterm survival in breast carcinomas. Br J Cancer 96: 801-807, 2007.

22. Sassen A, Diermeier-Daucher S, Sieben M, et al: Presence of HER4 associates with increased sensitivity to Herceptin in patients with metastatic breast cancer. Breast Cancer Res 11: R50, 2009.

23. Rodríguez-Antona $\mathrm{C}$, Leskelä $\mathrm{S}$, Zajac $\mathrm{M}$, et al: Expression of CYP3A4 as a predictor of response to chemotherapy in peripheral T-cell lymphomas. Blood 110: 3345-3351, 2007.

24. Kopp R, Rothbauer E, Ruge M, et al: Clinical implications of the EGF receptor/ligand system for tumor progression and survival in gastrointestinal carcinomas: evidence for new therapeutic options. Recent Results Cancer Res 162: 115-132, 2003.

25. Han SW, Oh DY, Im SA, et al: Phase II study and biomarker analysis of cetuximab combined with modified FOLFOX6 in advanced gastric cancer. Br J Cancer 100: 298-304, 2009.

26. Kim JG, Sohn SK, Chae YS, et al: TP53 codon 72 polymorphism associated with prognosis in patients with advanced gastric cancer treated with paclitaxel and cisplatin. Cancer Chemother Pharmacol 64: 355-360, 2009.

27. Huang ZH, Hua D and Du X: Polymorphisms in p53, GSTP1 and XRCC1 predict relapse and survival of gastric cancer patients treated with oxaliplatin-based adjuvant chemotherapy. Cancer Chemother Pharmacol (In press).

28. Viguier J, Boige V, Miquel C, et al: ERCC1 codon 118 polymorphism is a predictive factor for the tumor response to oxaliplatin/5-fluorouracil combination chemotherapy in patients with advanced colorectal cancer. Clin Cancer Res 11: 6212-6217, 2005.

29. Okuda K, Sasaki H, Hikosaka Y, et al: Excision repair cross complementation group 1 polymorphisms predict overall survival after platinum-based chemotherapy for completely resected non-small-cell lung cancer. J Surg Res (In press).

30. Kalikaki A, Kanaki M, Vassalou H, et al: DNA repair gene polymorphisms predict favorable clinical outcome in advanced non-small-cell lung cancer. Clin Lung Cancer 10: 118-123, 2009. 